\title{
Weighted and unweighted network of amino acids within protein
}

\author{
Md. Aftabuddin and Sudip Kundu* \\ Department of Biophysics, Molecular Biology 83 Genetics \\ University of Calcutta, 92, A.P.C. Road, Kolkata- 700009, India
}

(Dated:)

\begin{abstract}
The information regarding the structure of a single protein is encoded in the network of interacting amino acids. Considering each protein as a weighted and unweighted network of amino acids we have analyzed a total of forty nine protein structures that covers the three branches of life on earth. Our results show that the probability degree distribution of network connectivity follows Poisson's distribution; whereas the probability strength distribution does not follow any known distribution. However, the average strength of amino acid node depends on its degree $(\mathrm{k})$. For some of the proteins, the strength of a node increases linearly with $\mathrm{k}$. On the other hand, for a set of other proteins, although the strength increases linaerly with $\mathrm{k}$ for smaller values of $\mathrm{k}$, we have not obtained any clear functional relationship of strength with degree at higher values of $\mathrm{k}$. The results also show that the weight of the amino acid nodes belonging to the highly connected nodes tend to have a higher value. The result that the average clustering coefficient of weighted network is less than that of unweighted network implies that the topological clustering is generated by edges with low weights. The ratio of average clustering coefficients of protein network to that of the corresponding classical random network varies linearly with the number $(\mathrm{N})$ of amino acids of a protein; whereas the ratio of characteristic path lengths varies logarithmically with $\mathrm{N}$. The power law behaviour of clustering coefficients of weighted and unweighted network as a function of degree $\mathrm{k}$ indicates that the network has a signature of hierarchical network. It has also been observed that the network is of assortative type.
\end{abstract}

PACS numbers: 87.14.Ee

Keywords: protein, weighted network, assortative mixing

\section{INTRODUCTION}

A large number of researchers has been attracted to shed light on the topology, growth and dynamics of different kind of networks since 1990s. Studies on a large number of networks such as food webs, electrical power grids, the world wide web (WWW), coauthorship and citation network of scientists have been accelerated after the major breakthrough of Barabasi-Albert's work on the mapping of WWW and the physical internet in the late 1990s [1-6]. Like all branches of science, network analysis is increasingly recognised as a powerful approach to understanding of even the biological organisation and the function of cellular components and may also help us to understand the principles driving the evolution of living organism [7]. One could think the civilisation as a network of human and ecosystems. Whereas ecosystems are interaction of different organisms. Multicellular organism is a network of unicellular organisms. Even the cell is nowadays considered as a outcome of interactions of complex networks of genome, transcriptome, proteome, metabolome. Efforts have also been made to study the protein-protein interaction network [8-12] and also the amino acid network within protein [13-17]. Network of amino acids within a protein shows the 'small world' property, i.e, it is possible to connect any two amino acids of a protein through just a few links. It has also been observed that long range interactions of amino acids have scale free properties, i.e, they have a distribution of connectivities that decays with a power law tail. The scale free networks emerge in the context of growing network in which new vertices connect preferentially to the more highly connected vertices in the network. On the other hand, the short range interactions follow a Poisson like distribution, a characteristic of so called 'random network' which emerges as the result of random link within vertices. Recently, we have studied the networks of hydrophobic and hydrophilic amino acids within a protein separately [17] and have observed the small world properties for both the cases. Our studies also show that the average degree of the hydrophobic node is larger than the average degree of hydrophilic node. In all these studies protein has been considered as an unweighted network. Very recently, Barat et al [18] have proposed a framework of understanding the weighted network and have analysed the traffic and coauthorship networks. Amino acid network within a protein can be one of the best examples of the weighted network. In this paper, we have applied the concepts of weighted network to analyze and understand the native protein structures and their different network properties.

*Electronic address: skbmbg@caluniv.ac.in 


\section{METHODS}

\section{A. Protein as a weighted network}

Protein molecule is a polymer of twenty different amino acids joined by peptide bonds. The selection of specific amino acids is crucial for the molecule's native structure. Amino acids in different regions form local regular secondary structure ( $\alpha$ - helix, $\beta$ - sheet, etc.), which pack to form tertiary structure (active conformation) that in turn stabilizes three dimensional conformations. Several polypeptide chains forming the tertiary structure arrange to form quarternary structure (homo/hetero dimer complexes) [19]. In active 3-D conformational space of a protein, the amino acids may have different interactions with each other. In fact, the i-th amino acid of a protein may come in close contact with $(\mathrm{i}+\mathrm{j})$-th amino acid, where $\mathrm{j}$ may take a large value. Further, between any two amino acids the atoms of the side chains of two different amino acids may have the possibilities of more than one interaction. In the amino acid network within a protein, the amino acids are considered as nodes. A link between any two amino acids is considered to be present if any two atoms from two different amino acids are within $5 \AA$ distance (which is the higher cut-off of London-van der Waals forces [20]). Since several atoms of any amino acid 'i' may be within the prescribed distance of several atoms of another amino acid ' $\mathrm{j}$ '; there is a possibility of multiple links between different amino acids. These multiple links between any two different amino acids are the basis of the weight of the connectivity. The intensity $w_{i j}$ of the interaction between two amino acids ' $\mathrm{i}$ ' and ' $\mathrm{j}$ ' is defined as the number of possible links between $\mathrm{i}$ and $\mathrm{j}$-th amino acids. To understand the nature of link we have analysed a total of forty-nine protein structures [Table I] of nine different fold types that almost covers all the major four protein classes (all $-\alpha$, all $-\beta$, mixed $\alpha / \beta$, mixed $\alpha+\beta$ ) corresponding to the three branches of life on earth (archaea, bacteria, eukariya) [21].

\section{B. Network Parameters}

The most elementary property of the connectivity of a network is the degere of its node. The degree of any node 'i' is represented by $k_{i}=\sum_{j} a_{i j}$. Here $a_{i j}$ is the element of the adjacency matrix of the graph, whose value is 1 if an edge connects a node ' $\mathrm{i}$ ' to the another node ' $\mathrm{j}$ ' and 0 otherwise. For a weighted network, one wants to calculate the strength of a node represented by $s_{i}=\sum_{j} a_{i j} w_{i j}$. Here $w_{i j}$ is the number of possible interactions between any two ('i' and ' $\mathrm{j}$ ') amino acids. This parameter actually is a better representation of a protein network since it actually represents the number of connectivity of an amino acid with all other amino acids. It should be mentioned that we have not considered the energy of any interaction. The spread in the strength of a node has been characterised by a distribution function $P(s)$; where $P(s)=N(s) / \sum N(s), N(s)$ being the number of nodes with strength 's'. On the other hand the probability of degree distribution is represented by $P(k)=N(k) / \sum N(k)$.

To examine if there is any 'small world' property in the network, one conventionally have to determine the two parameters- i) the characteristic path length $(L)$ and ii) the clustering coefficient $(C)$. The characteristic path length $L$ of a graph is the path length between two nodes averaged over all pairs of nodes. Traditionally the clustering coefficient $C_{i}$ of a node ' $\mathrm{i}$ ' is the ratio between the total number $\left(e_{i}\right)$ of the edges actually connecting its nearest neighbour and the total number of all possible edges between all these nearest neighbour $\left[k_{i}\left(k_{i}-1\right) / 2\right.$; if 'i' vertex has $k_{i}$ neighbours] and is given by $C_{i}=2 e_{i} / k_{i}\left(k_{i}-1\right)$. The clustering coefficient of the whole network is the average of all individual $C_{i}$ 's. For a random network having $\mathrm{N}$ number of nodes with average degree $\langle k\rangle$, the characteristic pathlength $\left(L_{r}\right)$ and the clustering coefficient $\left(C_{r}\right)$ have been calculated using the expression $L_{r} \approx \operatorname{lnN} / \ln \langle k\rangle$ and $C_{r} \approx\langle k>/ \mathrm{N}$ given in [2]. According to Watts \& Strogatz [2], if $L$ and $C$ values of a network are such that $C>>C_{r}$ and $L \geq L_{r}$; then that network is said to have the 'small world' property.

Combining the topological information with the weight distribution of the network, Barat et al have introduced an analogous parameter of $\mathrm{C}$ and that is known as weighted clustering coefficient, $C_{i}^{w}$. The weighted clustering coefficient is given by

$$
C_{i}^{w}=\frac{1}{s_{i}\left(k_{i}-1\right)} \sum_{j, h} \frac{w_{i j}+w_{i h}}{2} a_{i j} a_{i h} a_{j h}
$$

This weighted clustering coefficient, $C_{i}^{w}$ is a measure of the local cohessiveness that takes into account the importance of the clustered structure on the basis of amount of interaction intensity (number of possible interactions between amino acids) actually found on the local triplets.

To study the tendency for nodes in networks to be connected to other nodes that are like (or unlike) them, we have first calculated the Pearson correlation coefficient ( $\mathrm{r}$ ) of the degrees at either ends of an edge. For our undirected unweighted protein network this $\mathrm{r}$ value has been calculated using the expression suggested by Newman [21] and is 
given as

$$
r=\frac{N^{-1} \sum_{i} j_{i} k_{i}-\left[N^{-1} \sum_{i} 0.5\left(j_{i}+k_{i}\right)\right]^{2}}{N^{-1} \sum_{i} 0.5\left(j_{i}^{2}+k_{i}^{2}\right)-\left[N^{-1} \sum_{i} 0.5\left(j_{i}+k_{i}\right)\right]^{2}}
$$

Here $j_{i}$ and $k_{i}$ are the degrees of the vertices at the ends of the $\mathrm{i}$-th degree, with $\mathrm{i}=1, . . \mathrm{N}$. This parameter would help us to understand whether the network is assortative or disassortative type. For an unweighted network this could also be understood calculating the average nearest neighbor degree for different $k$ and comparing this $k_{n n}$ with the $k$ values. If $k_{n n}(k)$ is an increasing function of $k$ then the network is of assortative type. On the other hand, for a weigheted network Barat et al have modified the expression for $k_{n n, i}$ and suggested the equivalent weighted average nearest-neighbors degree $k_{n n, i}^{w}$ to be defined as

$$
k_{n n, i}^{w}=\frac{1}{s_{i}} \sum_{j=1}^{N} a_{i j} w_{i j} k_{j}
$$

\section{RESULTS \& DISCUSSIONS}

We have analyzed the network of forty-nine proteins listed in Table I. For each of all the proteins, all the amino acids are part of a single network, i.e., there is no isolated node present in any of the networks. For each of the 49 proteins we have calculated the average degree $\langle k\rangle$. The values vary from 8.23 to 11.01 . The average of the $\langle k\rangle$ values for all 49 proteins was found to be 9.40 with standard deviation 0.55 . We have also observed that the value of average degree does not depend on the network size (i.e., on the number of amino acids of the protein). Next we have studied the degree and strength distributions of the nodes within the network. For all the proteins we have studied, the probability distributions $P(k)$ are of Poisson's distribution type as is evident from Fig 1 . However, for each of the proteins the probability strength distribution $P(s)$ of the nodes exhibit no clear pattern as is shown in Fig 2 . This dissimilarity in the functional behaviour of $P(k)$ and $P(s)$ demands further investigations on the relationship between $\operatorname{strength}(s)$ and degree $(k)$ of the nodes.

To understand the relationship between the strength of a node with its degree we have further studied the average strength $s(k)$ as a function of $k$. We have observed that the strength of a vertex changes with its degree $k$ [Fig 3]. For some of the proteins, there is a linear relationship of $s(k)$ with $k$,i.e., the strength of a vertex is simply proportional to its degree. On the other hand, for a set of other proteins, although the strength increases linearly with $\mathrm{k}$ for smaller values of $\mathrm{k}$, we have not obtained any clear functional relationship of strength with degree at higher values of $\mathrm{k}$. One of the possible explanations is as follows. Consider an amino acid ' $\mathrm{i}$ ' having a small number of amino acids interacting with it [Fig 4]. In this case, it is possible to accomodate most of the atoms of side chains of all interacting amino acids within $5 \AA$ distance of the $\mathrm{i}$-th amino acid. Hence, for smaller $\mathrm{k}$, the strength of a vertex increases almost linearly with its degree. On the other hand, for larger $\mathrm{k}$, there are more number of interacting amino acids than in the previous case. In such case, most of the atoms of a section of interacting amino acids may not fall within the prescribed distance due to spatial constraints imposed by the surrounding amino acids. Next, we have plotted the average weight $\left(\left\langle w_{i j}\right\rangle\right)$ as a function of the end-point degree $\left(k_{i} k_{j}\right)$ in Fig 5 . The average weight fluctuates for the whole range of $k_{i} k_{j}$ values. However, from Fig 5 , it is clear that although there are local fluctuations, there is a tendency of the average weights to increase with their $k_{i} k_{j}$ values (as depicted from the inset figure of Fig 5 ). The result implies that the weight of amino acid nodes belonging to the highly connected nodes tend to have a higher value.

We next describe how the clustering coefficients for both weighted and unweighted networks vary with their degree $\mathrm{k}$. At the same time we examine whether the networks have the 'small world' property or not. We also test whether there is any dependency of average clustering coefficient and average path length on the network sizes.

As mentioned earlier, we have followed Watts and Strogatz prescription to verify whether the protein network exhibit 'small world' property or not. We have calculated the average clustering coefficient $\langle C\rangle$ and the characteristic path length $\langle L\rangle$ for each of the forty-nine proteins and their respective values $\left.\left.\left(<C_{r}\right\rangle \&<L_{r}\right\rangle\right)$ for the random network having the same $\mathrm{N}$ and $\langle k\rangle$. In the present study, the $\langle C\rangle$ values vary from 0.464 to 0.586 ; whereas the ratios $\left(p=\left\langle C>/<C_{r}>\right.\right.$ ) of average clustering coefficient to that of the corresponding classical random graph vary from 4.61 to 25.20 . On the other hand, the characteristic path length is of the same order as that of the corresponding random network. Although the ratios (p) for protein networks are not of the order of $10^{2}-10^{4}$ as observed in the case of scientific collboration networks and network of film actors, there are several other networks where ' $\mathrm{p}$ ' may have smaller values $[1,4-6,17]$. For example, the ratio for metabolic network, protein-protein interaction network, food webs and network of C.Elegans has values 5.0, 4.4, 12.0 and 5.6 respectively. The above results indicate the small world property of the protein network. 
From the above examples, it is clear that the ratio (p) varies from a smaller value to a very large value. In our protein networks, the ratio (p) also varies in a wide range. Moreover it is also observed that the ratios (q) of $\langle L\rangle$ and $\left\langle L_{r}\right\rangle$ are not constant. We have further studied the dependencies of $\mathrm{p}$ and $\mathrm{q}$ on $\mathrm{N}$ [Fig 6$]$. It has been observed that both the ratios $\mathrm{p}$ and $\mathrm{q}$ vary with the number of nodes (i.e., the number of amino acids); but with a different relationships. The ratio $(\mathrm{p})$ of clustering coefficients varies linearly with the number $(\mathrm{N})$ of amino acids of the protein; whereas the ratio (q) of characteristic path lengths varies logarithmically with N.

We have already mentioned that the average clustering coefficient of the protein networks varies from 0.464 to 0.586 . However, the average weighted clustering coefficient values for the same set of protein networks vary from 0.249 to 0.325. The values of $\langle C\rangle$ and $\left\langle C_{w}\right\rangle$, when averaged over all of the forty-nine proteins we have studied, are 0.511 and 0.269 respectively [Table II]. And for each of the proteins, the $\left\langle C_{w}\right\rangle$ value is always less than that of $\langle C\rangle$. It implies that the topological clustering is generated by edges with low weight. The results also indicate that the largest part of interactions (i.e., interactions between two amino acids ) is occuring on edges (amino acids) not belonging to interconnected triplets. Therefore the clustering has a minor effect in the organization of amino acid network within protein.

Next we are interested to study if there is any hierarchy in the amino acid network. In a hierarchical network, the low degree nodes belong generally to well interconnected communities (high clustering coefficients) with hubs connect many nodes that are not directly connected (small clustering coefficient). It has been stated that the signature of the hierarchical modularity lies in the scaling coefficient of $C(k) \sim k^{-\beta}$, when $\beta$ has a value of 1 ; whereas, for a non-hierarchical network the value of $\beta$ is 0 [6-7]. For the amino acid network within proteins, both the $C(k)$ and $C^{w}(k)$ exhibit a power-law decay as a function of $k$ as is evident from Fig 7 . The scaling coefficient $(\beta)$ for the $C(k)$ varies from 0.247 to 0.367 with an average of 0.322 ; whereas the corresponding coefficient $\left(\beta_{1}\right)$ for $C^{w}(k)$ varies from 0.525 to 0.722 with an average of 0.637 [Table II]. First of all, we get a power law decay for both $\mathrm{C}(\mathrm{k})$ and $C^{w}(k)$, but the values of the scaling coefficients lie within the range of 0 and 1 . The values of the scaling coefficients imply that the networks have a tendency of hierarchical nature. Surprisingly, the values of $\langle C\rangle$ are nearly double that of $\left\langle C_{w}\right\rangle$, but the average value of $\beta$ is nearly half that of $\beta_{1}$. The result implies that topological clustering is generated by amino acid edges with low weights. Moreover, for smaller $k$, the amino acids within a cluster are linked through higher weights than those related with larger $k$ values. For larger $k$ values, due to spatial constrains imposed by neighboring amino acids within the cluster, the nodes (amino acids) have less number of interactions between them. As $\mathrm{k}$ increases, the number of possible interactions (weights) among amino acids within the cluster decays faster than their connectivities [Fig 4].

We have also calculated the Pearson Correlation coefficients ( $r$ ) for each of the protein network. The $r$ values vary from 0.18 to 0.38 with an average of 0.26 and standard deviation 0.05 [Table II]. The positive $\mathrm{r}$ values suggest the assortative mixing behaviour of the nodes of the network [21]. In a protein, the amino acids with high degree have a tendency to be attached with the amino acids having high $\mathrm{k}$ values. We have also calculated the average degree of nearest neighbors for both unweighted and weighted networks. For each of the proteins, both the weighted and unweighted nearest neighbor values increase intially with increment of $\mathrm{k}$, but for a larger $\mathrm{k}$ there is a tendency of saturation [Fig 8], which might be explained as the steric hindrance of connecting amino acids due to three dimensional structural organisation of the protein. Because of such steric hindrance, the position of any amino acid in $3 \mathrm{D}$ conformational space is restricted resulting in the maximum values of degree $\left(k_{\max }\right)$ and strength $\left(s_{\max }\right)$ of a node. In fact, all these network properties are mainly governed by two major important factors - (i) the movement of any i-th amino acid is restricted by (i-1)-th and (i+1)-th amino acids through peptide bonds; and (ii) the attachment of an amino acid to any other amino acid does not only depend on their physico-chemical properties, but is also restricted by the spatial constraints imposed by neighboring amino acids, i.e., the preferential attachment is restricted by a maximum cut-off value due to steric hindrance. It should be mentioned that Amaral et al have discussed about the constraints limiting the addition of new links and argued that the nature of such constraints may be the controlling factor for the emergence of different classes of networks [23]. Hence, there is a need of new model to understand the phenomenology behind the network of a polymer in three dimensional space.

\section{SUMMARY}

In summary, the amino acid network within a protein has a small world property. The probability degree distribution of the amino acid network connectivity within protein is of Poisson's distribution type; whereas the probability strength distribution does not follow any particular pattern. We have further observed that the strength of a node changes with its degree $\mathrm{k}$. The ratios of the clustering coefficients of the protein network and its corresponding random network vary linearly with the number(N) of amino acids of the protein; while the respective ratios for characteristic path lengths vary logarithmically with $\mathrm{N}$. The results further indicate that the topological clustering is genereated by amino acids (edges) with low weights. The amino acid networks within protein exhibit the signature of a hierarchical 
network. The protein network is an outcome of assortative mixing of the amino acid nodes.

\section{Acknowledgments}

The authors acknowledge the computational support provided by Distributed Information Center of Calcutta University. The authors also thankful to Prof. U. Chaudhuri and Dr. G. Gangopadhyay for their constant inspirations.

[1] J.M. Montoya and R.V. Sole, J. Theor. Biol. 214, 405 (2002)

[2] D.J. Watts and S.H. Strogatz, Nature 393, 440 (1998)

[3] A.L. Barabasi and R. Albert, Science 286, 509 (1999) and T. Vicsek, Physica A 311, 590 (2002)

[4] A.L. Barabasi, et al. Physica A. 311, 590 (2002)

[5] M.E.J. Newman, Proc. Natl. Acad. Sci. USA 98, 404 (2001)

[6] E. Ravsaz, et al. Science 297, 1551 (2002)

[7] A.L. Barabasi and Z.N. Oltavi, Nature Review Genetics 5, 101 (2004)

[8] A. Wagner, Mol. Biol. Evol. 18, 1283 (2001)

[9] A. Wagner and D.A. Fell, Proc. R. Soc. London B. 268, 1803 (2001)

[10] H. Jeong, et al. Nature 411, 41 (2001)

[11] S. Wutchy, Mol. Biol. Evol. 18, 1694 (2001)

[12] H. Jeong, et al. Nature 407, 651 (2000)

[13] M. Vendruscolo, et al. Phys. Rev. E 65, 061910 (2002)

[14] N.V. Dokholyan, et al. Proc. Natl. Acad. Sci. USA 99, 8637 (2002)

[15] J. Qian, N.M. Luscombe, and M. Gerstein, J. Mol. Biol. 313, 673 (2001)

[16] L.H. Greene and V.A. Higman, J. Mol. Biol. 334, 781 (2003)

[17] S. Kundu, Physica A 346, 104 (2005)

[18] A. Barat, et al. Proc. Natl. Acad. Sci. USA 101, 3747 (2004)

[19] C. Brandstein and T. Tooze, Introduction to Protein structure, Garland Publisher, New York (1999)

[20] I. Tinoco (Jr.), K. Sauer, and J.C. Wang, Physical Chemistry: Principles and Application in Biological Sciences, PrinticeHall, New Jersey, 456-544

[21] PDB: Protein data bank, http://www.rcsb.org/

[22] M.E.J. Newman, Phys. Rev. Lett. 89, 208701 (2002)

[23] L.A.N. Amaral, et al. Proc. Natl. Acad. Sci. USA 97, 11149 (2000) 
TABLE I: List of PDB Ids of the proteins used in our study.

\begin{tabular}{|c|c|c|c|c|c|c|c|c|c|}
\hline 1AVA_c & 1CE7_b & 1EYL_a & 1HWN_b & 1JLY_b & 2AAI_b & $1 \mathrm{WBA}$ & 7TIM_a & 1AGD_b & 1BIH_a \\
\hline 1CD8 & 1EPF_d & 1HYX $\_$ & 1TLK & 3KBP_c & 1HNG_a & 1B8A_a & 1E86_a & 1FAP_b & 1EOV_a \\
\hline $1 \mathrm{GVP}$ & 1MJC & 1H7I_a & 1JR8_a & 1PSD_a & 1RIS & 1URN_c & $2 \mathrm{ACY}$ & 1VLT_a & 1AYC_a \\
\hline 256B_a & 1CJ1_a & 1FBV_a & 1SHA_a & 2HMZ_a & 1BEB_a & 1BJ7 & 1GM6_a & 1OBP_a & 1RPX_a \\
\hline 1BMT_a & 1DIO_a & $1 \mathrm{PDO}$ & 3ECA_a & 3RAB_a & 1EUN_a & 1G4T_a & 1HO4_a & 1NSJ & \\
\hline
\end{tabular}

TABLE II: The average clustering coefficient of weighted $\left.\left(<C_{w}\right\rangle\right)$ and unweighted $(<C>)$ network; the scaling coeffeicients of power law decay of clustering coeffients as a function of degree $\mathrm{k}$ and Pearson correlation coefficients of the protein networks.

\begin{tabular}{ccccc}
\hline$\langle C\rangle$ & $\left\langle C_{w}\right\rangle$ & $C(k) \sim k^{-\beta}$ & $C_{w}(k) \sim k^{-\beta_{1}}$ & $\mathrm{r}$ \\
& Value for $\beta$ & Value for $\beta_{1}$ & \\
\hline $0.511 \pm 0.026$ & $0.269 \pm 0.023$ & $0.322 \pm 0.023$ & $0.637 \pm 0.034$ & $0.259 \pm 0.049$ \\
\hline
\end{tabular}

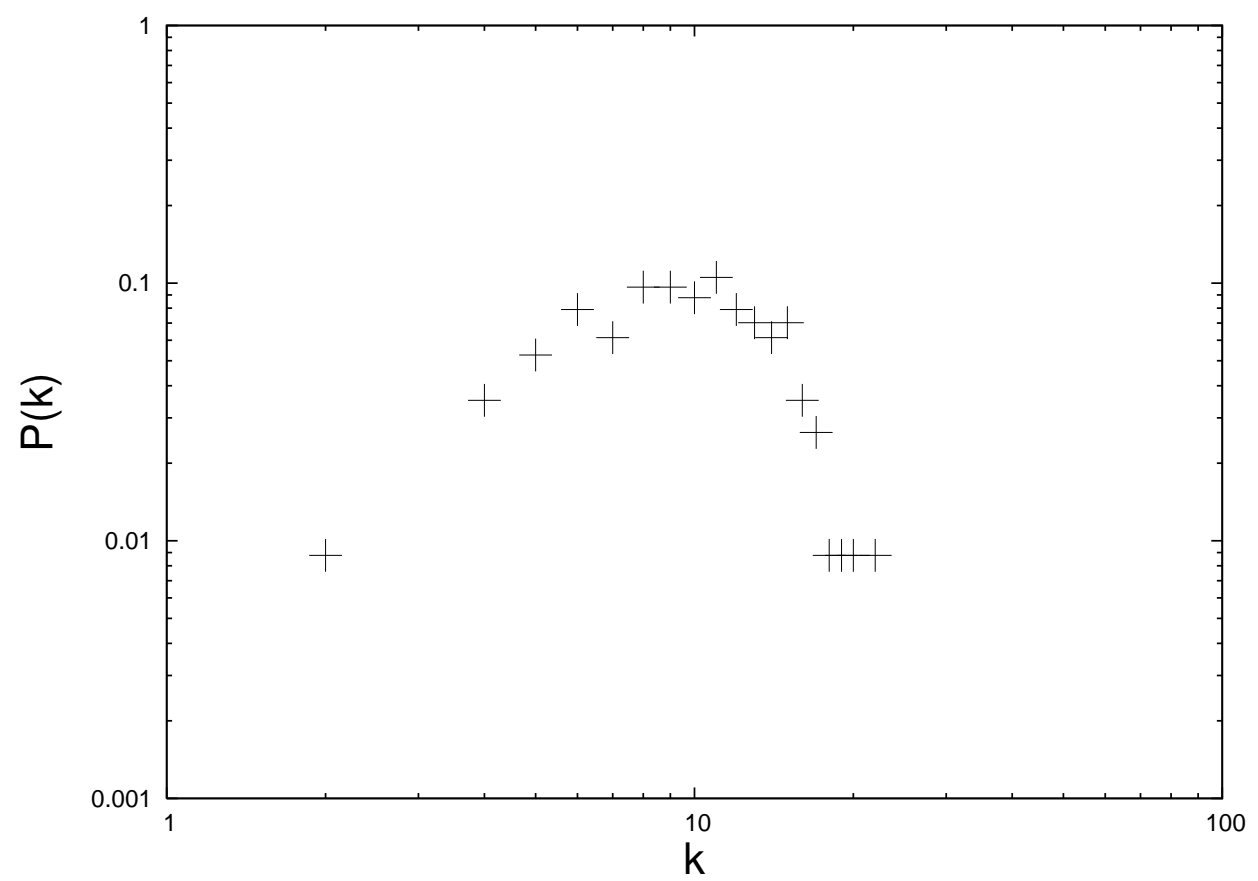

FIG. 1: A representative plot (PDB Id: 1CD8) of probability degree distribution of amino acid network within protein. The degree $\mathrm{k}$ of any amino acid ' $\mathrm{i}$ ' is the number of amino acids interacting with i. The distribution follows Poisson's distribution. 


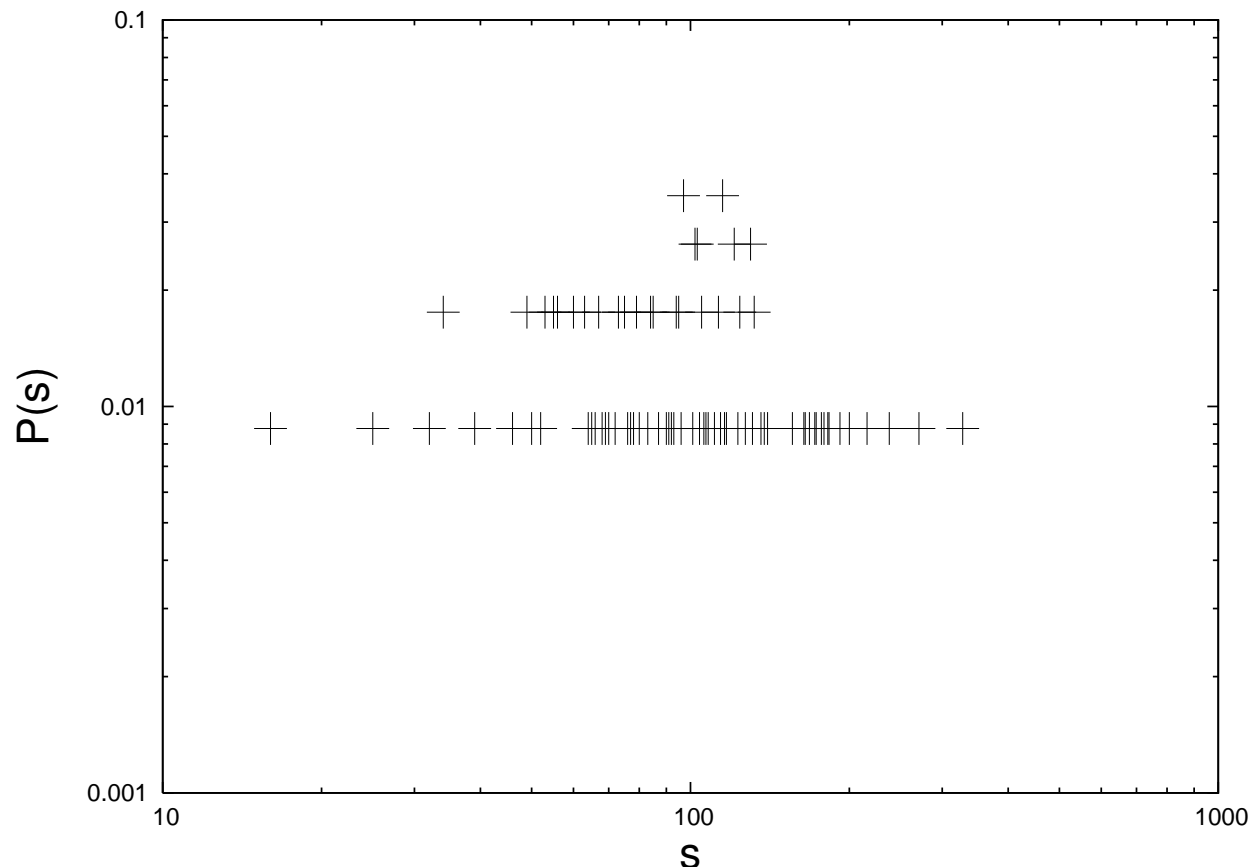

FIG. 2: A representative plot (PDB Id:1CD8) of probability strength distribution. The distribution has no definite functional form.

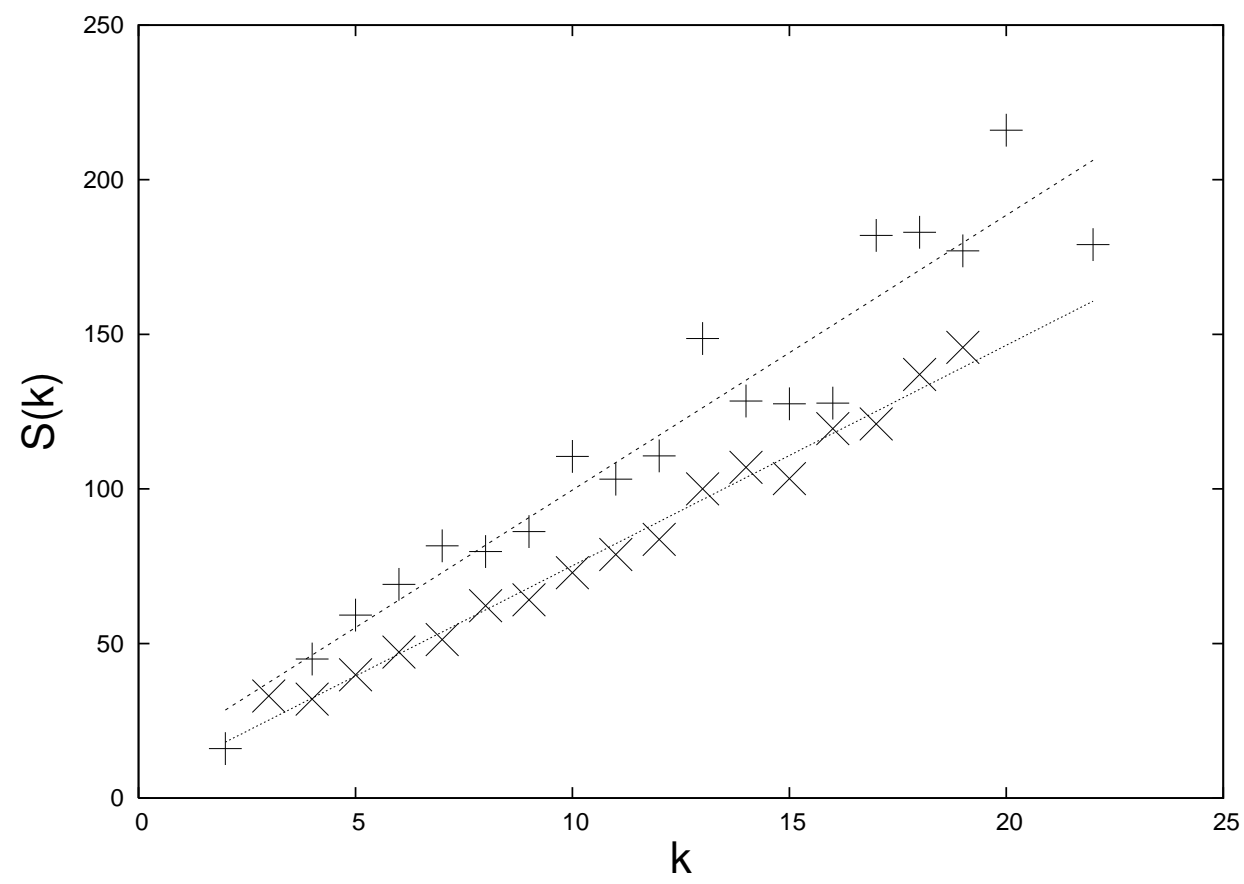

FIG. 3: A representative plot (PDB Id:1CD8, shown by plus sign and PDB Id:3KBP_c, shown by cross sign) of average strength $\mathrm{s}(\mathrm{k})$ as function of degree $\mathrm{k}$ of nodes. 


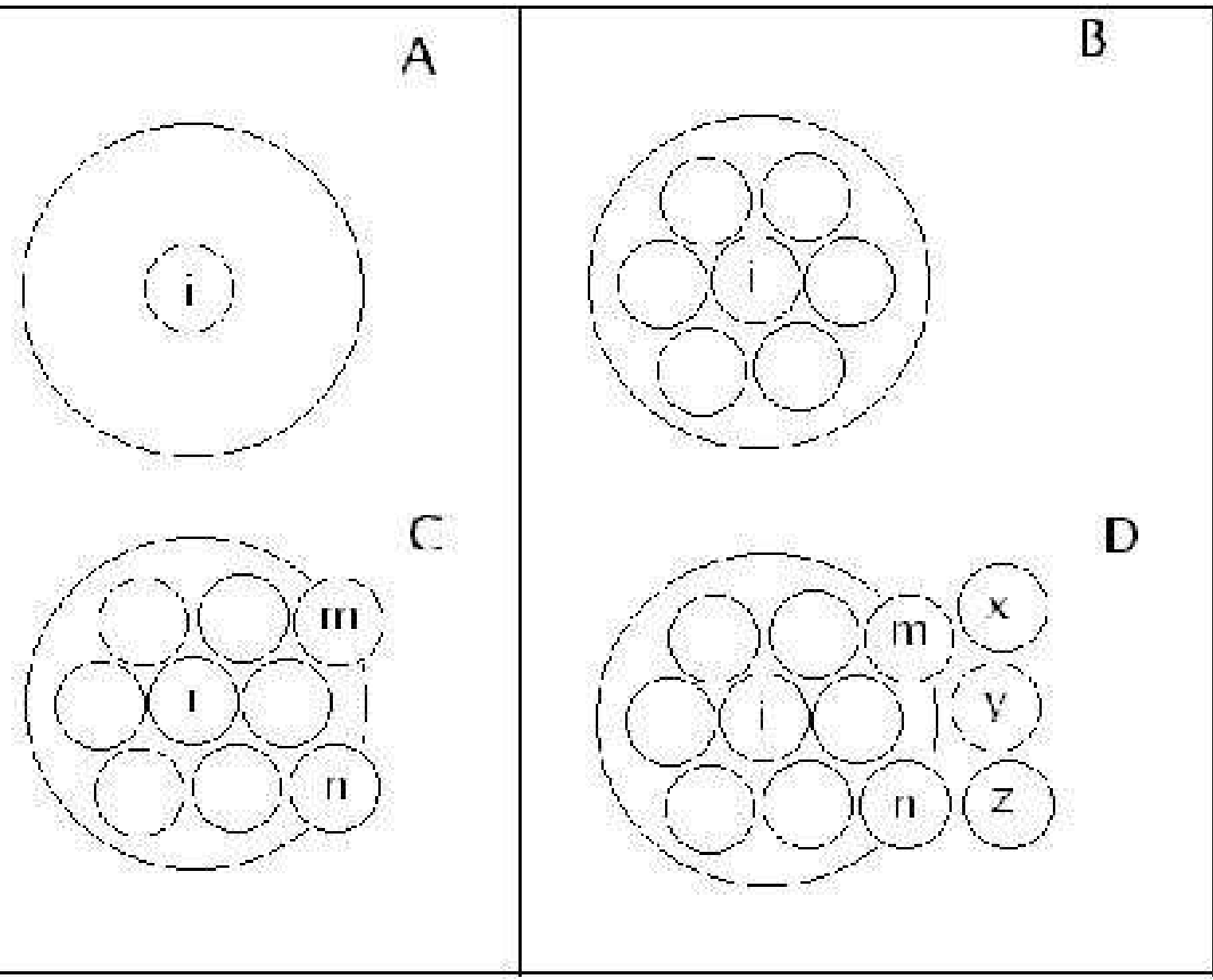

FIG. 4: Schematic diagram of (A) An amino acid 'i' in conformational space. The large circle represents the $5 \AA$ distance from the line representing the i-th amino acid. Any amino acid within that large circle should have a possible interaction (link) with the i-th amino acid. (B) Atoms of all amino acids are within $5 \AA$ distance of i-th amino acid. (C) Some of the atoms of a section of amino acids (marked as 'm' and ' $n$ ') are not within that large circle. All the amino acids have a possible link with the i-th amino acid. All the atoms of all amino acids, except two amino acids (marked as ' $m$ ' and ' $\mathrm{n}$ ') have possible interactions with i-th amino acid. However, a section of atoms (outside the large circle) of the two amino acids, 'm' and ' $n$ ', have no interaction with any of the atoms of i-th amino acid. (D) Three amino acids, ' $x$ ', ' $y$ ' and ' $z$ ', are completely outside the large circle and hence do not have any possible interaction with the i-th amino acid. 


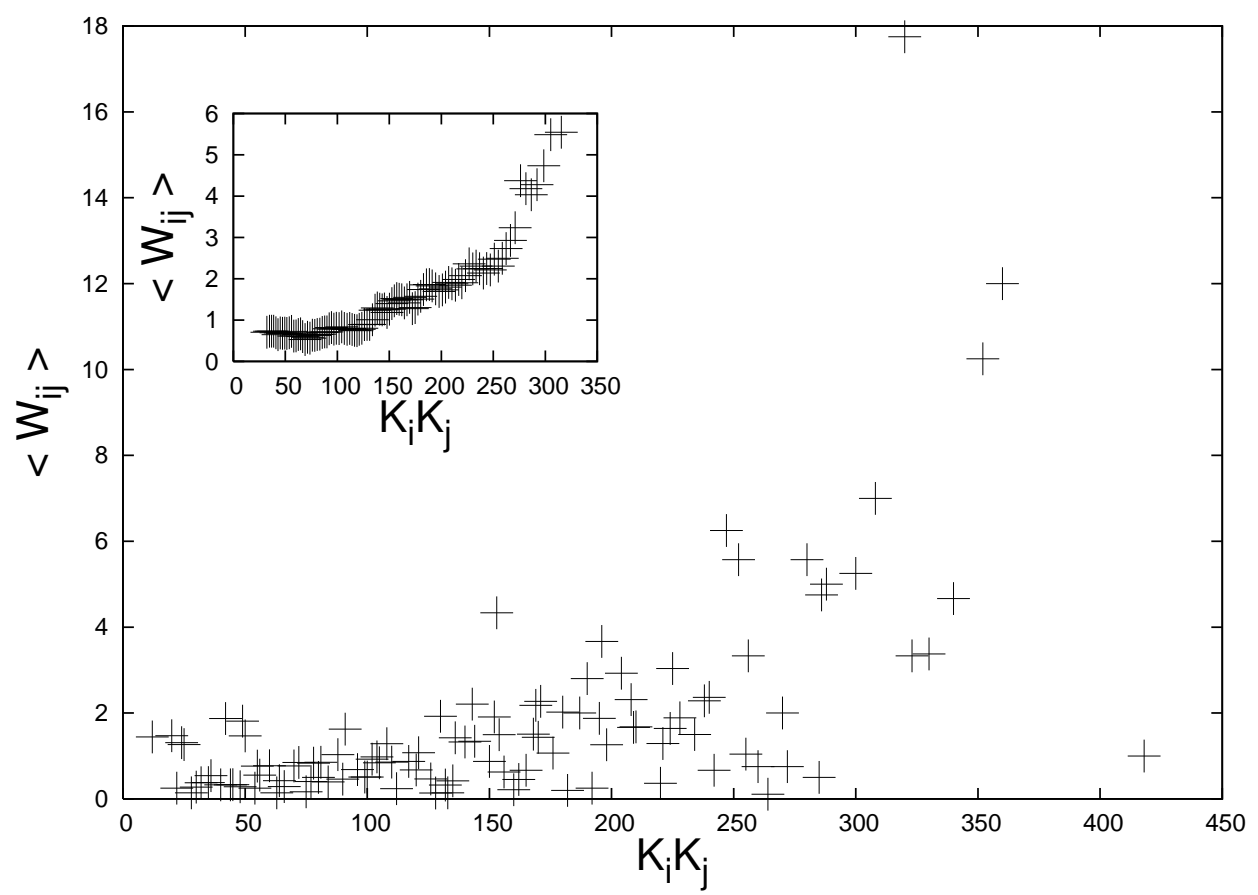

FIG. 5: A represntative figure (PDB Id:1CD8) of average weight as a function of the end-point degree. The running average of end-point degree with an window size of 15 is shown in inset.

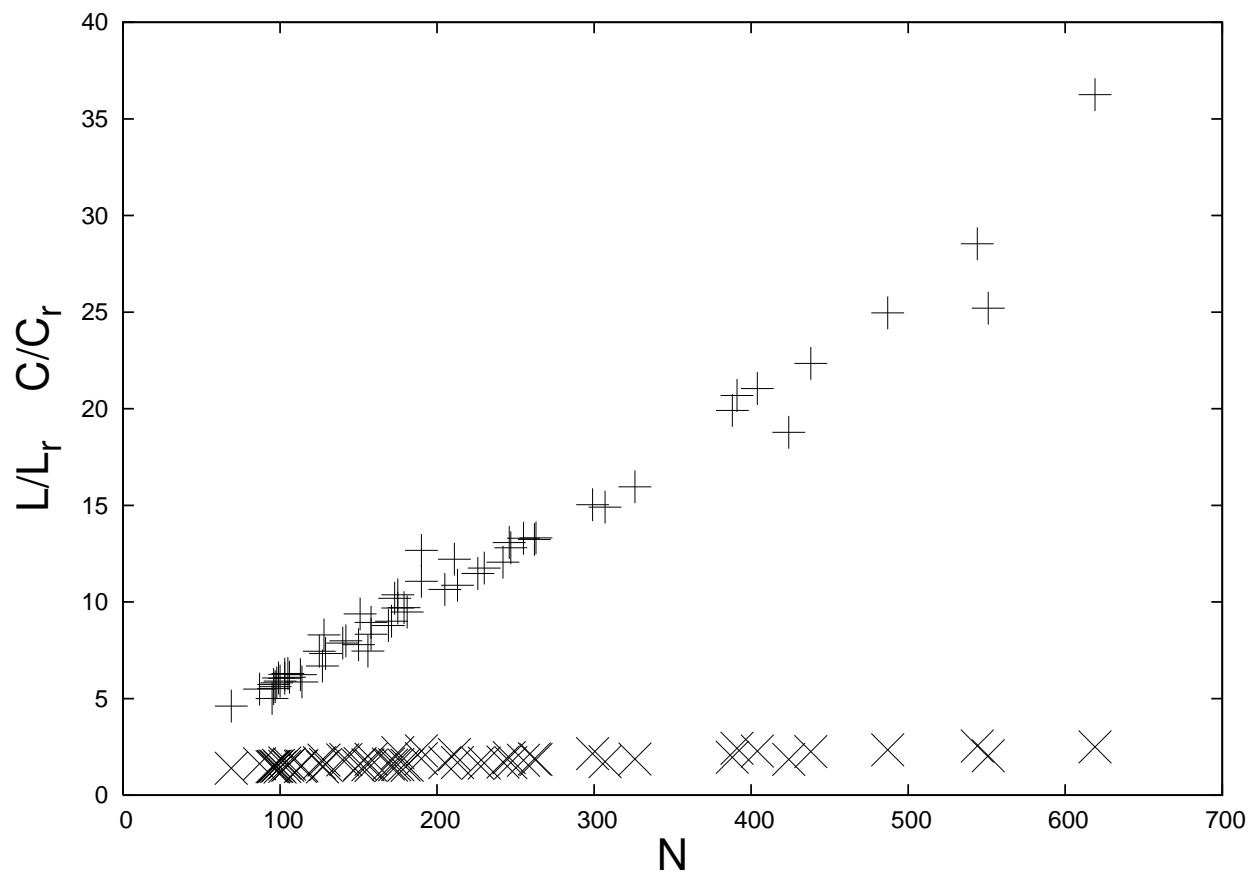

FIG. 6: The ratios $\mathrm{p}\left(=<C>/<C_{r}>\right)$ and $\mathrm{q}\left(=<L>/<L_{r}>\right)$ as a function of network size N. The ratio $\mathrm{p}$ (shown by plus sign) varies linearly with $\mathrm{N}$; whereas the ratio q (shown by cross sign) varies logarothmically with $\mathrm{N}$. 


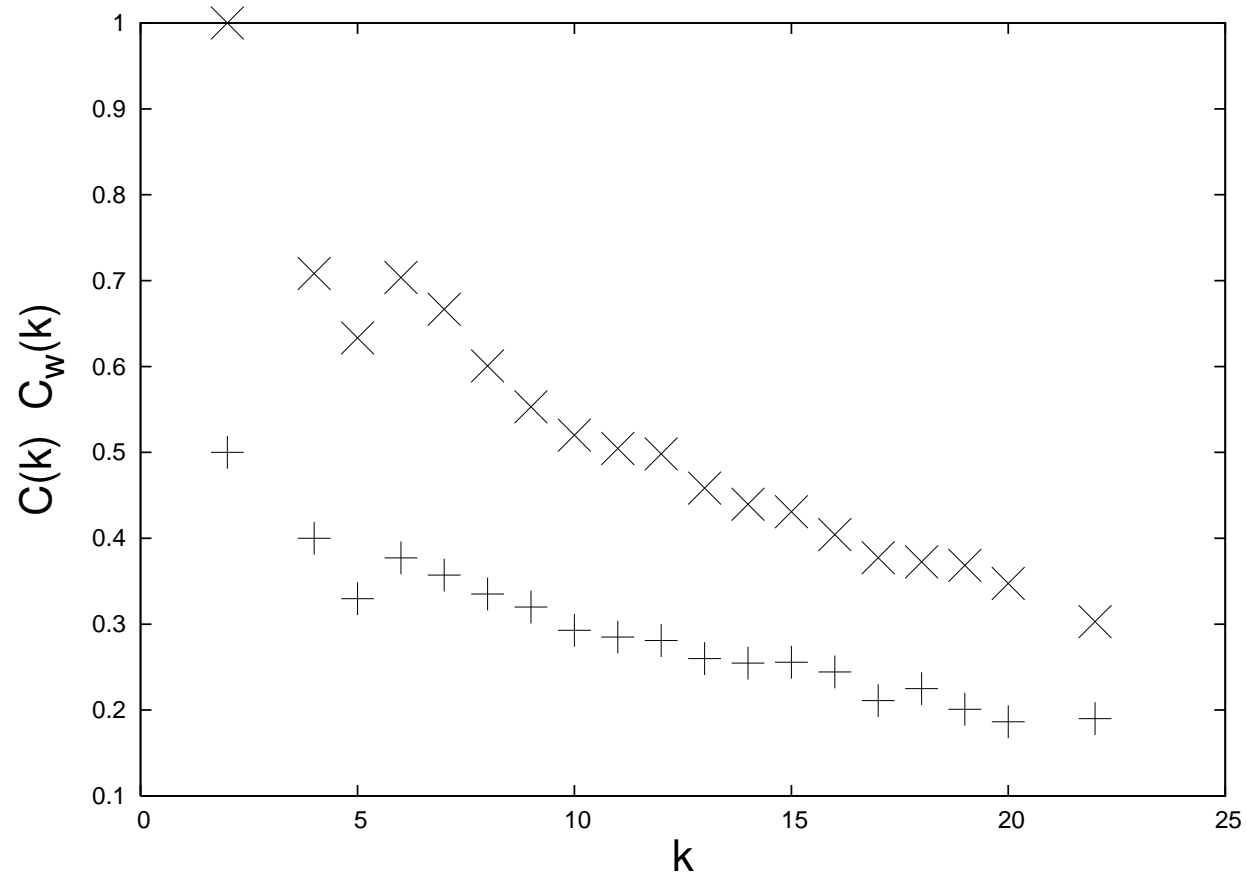

FIG. 7: A representative figure (PDB Id:1CD8) of topological clustering coefficient C(k) (shown by cross sign) and weighted clustering coefficient $C_{w}(k)$ (shown by plus sign) as a function of degree k of nodes.

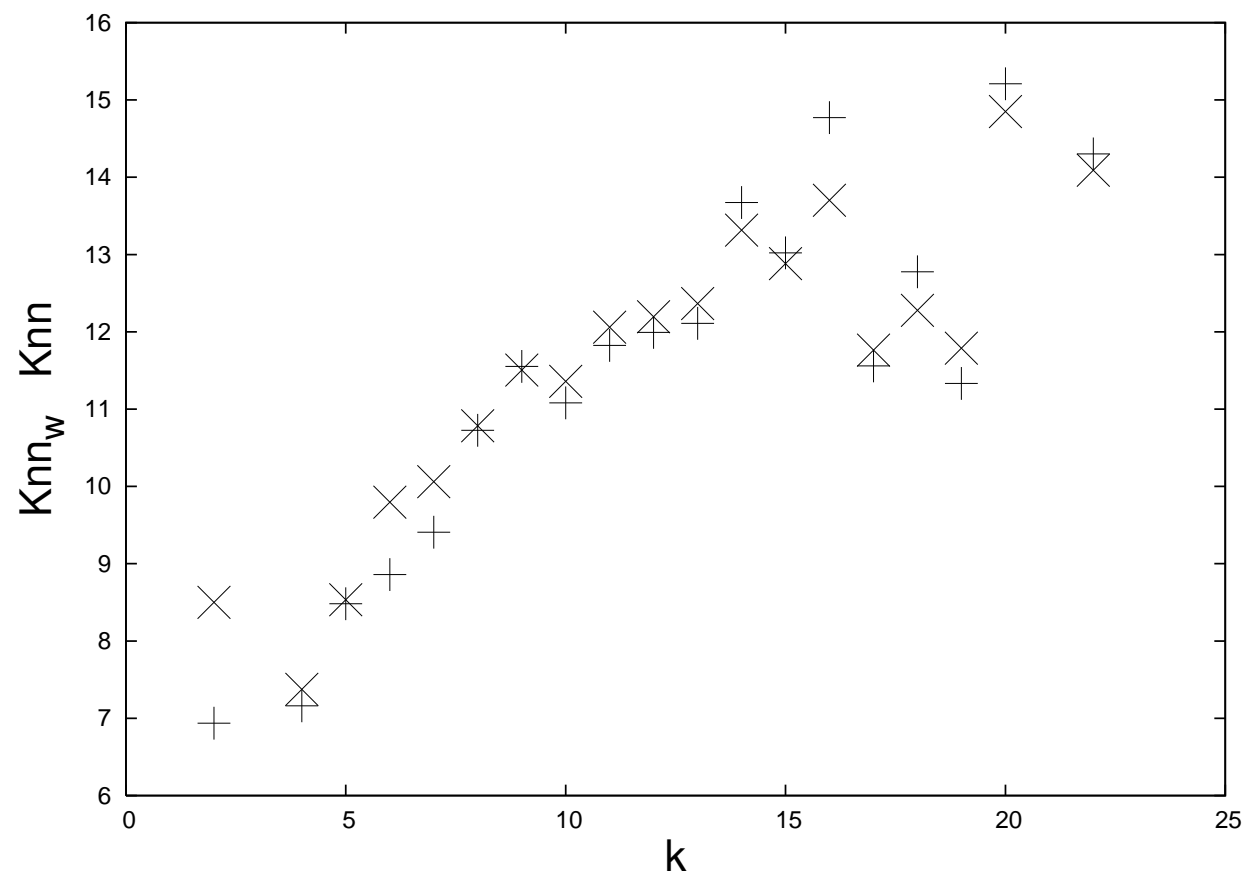

FIG. 8: A represented figure (PDB Id:1CD8) of unweighted $K_{n n}$ (shown by cross sign) and weighted $K_{n n}(w)$ (shown by plus sign) average degree of nearest neighbor as a function $\mathrm{k}$. 